\title{
The Effects of Short-Term Norepinephrine Up-Titration on Hemodynamics in Cardiogenic Shock
}

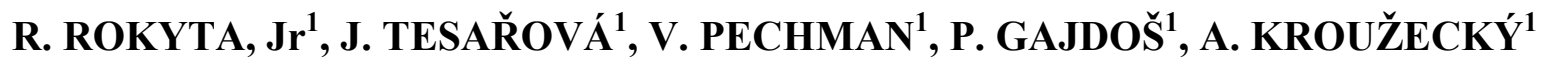 \\ ${ }^{1}$ Intensive Cardiac Care Unit, Department of Medicine I, Faculty of Medicine Plzeň, Charles \\ University Prague, Plzeň, Czech Republic
}

Received April 8, 2009

Accepted June 23, 2009

On-line August 12, 2009

\begin{abstract}
Summary
A higher mean arterial pressure (MAP) achieved by norepinephrine up-titration may improve organ blood flow in critically ill, whereas norepinephrine-induced afterload rise might worsen myocardial function. Our aim was to assess the effects of norepinephrine dose titration on global hemodynamics in cardiogenic shock. We prospectively evaluated 12 mechanically ventilated euvolemic patients (aged $67 \pm 12$ years) in cardiogenic shock (10 patients acute myocardial infarction, 1 patient dilated cardiomyopathy, 1 patient decompensated aortic stenosis). Hemodynamic monitoring included arterial and Swan-Ganz catheters. The first data were obtained at MAP of $65 \mathrm{~mm} \mathrm{Hg}$, then the norepinephrine dose was increased over $40 \mathrm{~min}$ to achieve MAP of $85 \mathrm{~mm} \mathrm{Hg}$. Finally, the norepinephrine-dose was tapered over $40 \mathrm{~min}$ to achieve MAP of $65 \mathrm{~mm} \mathrm{Hg}$. Norepinephrine up-titration increased MAP to the predefined values in all patients with concomitant mild increase in filling pressures and heart rate. Systemic vascular resistance increased, whereas cardiac output remained unchanged. During norepinephrine down-titration, all hemodynamic parameters returned to baseline values. We observed no changes in lactate levels and mixed venous oxygen saturation. Our data suggest that short-term norepinephrine dose up-titration in cardiogenic shock patients treated or pretreated with inotropes was tolerated well by the diseased heart.
\end{abstract}

\section{Key words}

Norepinephrine - Cardiac function - Shock - Hemodynamics • Pressure

\section{Corresponding author}

Richard Rokyta Jr, Intensive Cardiac Care Unit, Department of Medicine I, Faculty of Medicine, Charles University, Alej svobody
80, 32300 Plzen, Czech Republic. Fax: +420 377103163. E-mail: rokyta@fnplzen.cz

\section{Introduction}

Neurohumoral activation, including increased endogenous catecholamine levels (norepinephrine and epinephrine), is a hallmark of pathophysiological condition in chronic heart failure patients and it has several long-term harmful effects (Francis 1985). Conversely, exogenous catecholamines are widely administered to well volume-resuscitated critically ill patients in various clinical scenarios. The primary aim of this treatment is the preservation of adequate organ blood flow keeping mean arterial pressure (MAP) above the autoregulatory organ perfusion pressure threshold (Albanese et al. 2004, Matějovič 2005, Durišová et al. 2008). In physiological conditions, the organ blood flow is usually preserved over a wide MAP range. When MAP is less than $65 \mathrm{~mm} \mathrm{Hg}$, the organ blood flow may be compromised. In certain subgroups of intensive care unit patients (e.g. patients with preexisting arterial hypertension, after cardiopulmonary resuscitation, sepsis etc.), the optimal target value of MAP may be even higher than usually accepted values of $65-70 \mathrm{~mm} \mathrm{Hg}$ (i.e. above 80-90 mm Hg) (Matějovič 2005, Marik 2004, Tamborini et al. 2001). The influence of norepinephrine dose up-titration (resulting in a higher MAP usually within physiological range) on global and regional hemodynamics in sepsis (high cardiac output at baseline) has been assessed in several experimental and clinical studies showing no detrimental effects on global hemodynamics or cardiac function (Bourgoin et al. 2005, 
LeDoux et al. 2000, Di Giantomasso et al. 2003, Kroužecký et al. 2006). However, such an approach might worsen myocardial performance in critically ill patients with a failing or injured heart due to norepinephrine induced rise in afterload (Heusch 1990, de Zeeuw et al. 2001). To our knowledge, hemodynamic studies on MAP changes within physiological range induced by norepinephrine titration in patients with acute forms of ischemic heart disease or low cardiac output states are lacking. Therefore, we have conducted a prospective clinical trial to assess the short-term effects of various norepinephrine doses (leading to clinically significant MAP changes) on global hemodynamics in cardiogenic shock patients.

Table 1. Patients' characteristics.

\begin{tabular}{|c|c|c|c|c|c|c|c|}
\hline Pt. & Age & Diagnosis & $\begin{array}{c}\text { LVEF/RVEF } \\
(\%)\end{array}$ & MR & $\begin{array}{c}\text { APACHE II } \\
\text { score }\end{array}$ & $\begin{array}{l}\text { SOFA } \\
\text { score }\end{array}$ & $\begin{array}{c}\text { ICCU } \\
\text { survival }\end{array}$ \\
\hline 1 & 79 & STEMI & $35 / 55$ & $2 / 4$ & 14 & 7 & 0 \\
\hline 2 & 78 & STEMI & $30 / 30$ & $1 / 4$ & 20 & 12 & 0 \\
\hline 3 & 75 & STEMI & $25 / 60$ & $2 / 4$ & 14 & 4 & 0 \\
\hline 4 & 73 & Non-STEMI & $30 / 50$ & $2 / 4$ & 16 & 8 & 0 \\
\hline 5 & 61 & DCMP & $25 / 35$ & $3 / 4$ & 13 & 12 & 0 \\
\hline 6 & 59 & STEMI & $25 / 55$ & $2 / 4$ & 9 & 6 & 1 \\
\hline 7 & 70 & Aortic stenosis & $20 / 45$ & $1 / 4$ & 24 & 10 & 1 \\
\hline 8 & 65 & STEMI & $40 / 55$ & $2 / 4$ & 22 & 6 & 1 \\
\hline 9 & 32 & STEMI & $20 / 45$ & 0 & 20 & 9 & 1 \\
\hline 10 & 70 & STEMI & $30 / 50$ & $1 / 4$ & 23 & 7 & 1 \\
\hline 11 & 74 & Non-STEMI & $45 / 60$ & $1 / 4$ & 20 & 9 & 1 \\
\hline 12 & 68 & STEMI & $30 / 45$ & $1 / 4$ & 25 & 10 & 0 \\
\hline
\end{tabular}

LVEF/RVEF - left/right ventricular ejection fraction, MR - degree of mitral regurgitation, SOFA - sequential organ failure assessment, ICCU survival: 0 - no, 1 - yes, STEMI - ST elevation myocardial infarction, DCMP - dilated cardiomyopathy

\section{Methods}

The study followed the principles established in the Declaration of Helsinki. The protocol was accepted by the local ethics committee and informed consent was obtained from the patients or next of kin. This study was performed in intensive cardiac care unit in a tertiary medical center.

\section{Patients}

We evaluated 12 patients (age $67 \pm 12$ years, male/female $8 / 4$, for patients' characteristics see Table 1 ) fulfilling the following inclusion criteria: 1) pulmonary arterial occlusion pressure (PAOP) $\geq 15 \mathrm{~mm} \mathrm{Hg}, 2)$ the need for catecholamines to maintain MAP $\geq 65 \mathrm{~mm} \mathrm{Hg}$, 3) cardiac index $\leq 2.51 / \mathrm{min} / \mathrm{m}^{2}$, and 4) age $18-80$ years.

The exclusion criteria were major hemodynamic and/or electrical instability. All patients were mechanically ventilated (no ventilatory changes during the study) and sedated with midazolam and fentanyl.
Three patients had atrial fibrillation and 9 patients had sinus rhythm throughout the study. All but one patient with ST elevation myocardial infarction (STEMI) were treated with primary percutaneous coronary intervention (PCI) with stent implantation. Three patients were on intraaortic balloon counterpulsation. Six patients were given dobutamine in constant dosages $(2-10 \mu \mathrm{g} / \mathrm{kg} / \mathrm{min})$ during the study and 8 patients received levosimendan in previous days in the intensive care unit.

\section{Methods}

Hemodynamic monitoring included arterial and pulmonary arterial thermodilution catheters (Swan-Ganz hands-off infusion port catheter 7, $5 \mathrm{Fr}$, Arrow ${ }^{\circledR}$, Reading, USA). Both pulmonary arterial occlusion pressure (PAOP) and cardiac output (CO) were measured at end-expiration. Cardiac output was measured in triplicate at each time point $(10 \mathrm{ml}$ of room temperature saline, CO-set + closed injectate delivery system, Edwards Lifesciences, Unterschleissheim, Germany) and 


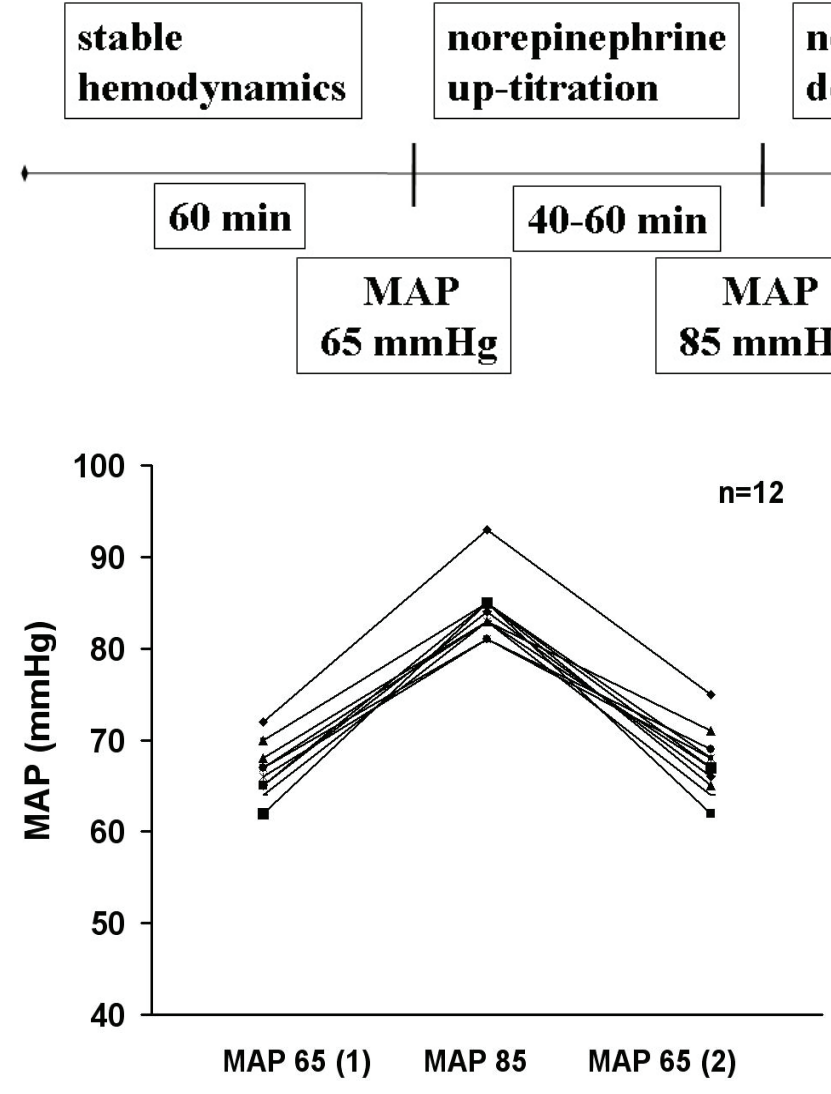

Fig. 2. Time course of mean arterial pressure. MAP - mean arterial pressure: individual changes

the mean of the three measurements was taken for calculations. Systemic and pulmonary vascular resistances (SVR and PVR) as well as left ventricular stroke work index (LVSWI) were calculated according to standard formulae. Blood gases were measured using a blood gas analyzer (ABL $520^{\circledR}$, Radiometer, Copenhagen, Denmark), and hemoglobin oxygen saturation was determined by a co-oximeter (OSM-3, Radiometer).

\section{Protocol (Fig. 1)}

Before the study, adequate fluid resuscitation was ensured on the individual basis by monitoring filling pressures (central venous pressure and pulmonary arterial occlusion pressure) and cardiac output with eventual subsequent fluid challenge. Afterwards, norepinephrine (NE) dose was titrated over approximately $40 \mathrm{~min}$ to achieve mean arterial pressure (MAP) $65 \mathrm{~mm} \mathrm{Hg}$ (at least $30 \mathrm{~min}$ in a steady state). At MAP of $65 \mathrm{~mm} \mathrm{Hg}$, the first data set was obtained, then NE dose was increased over $40 \mathrm{~min}$ to achieve MAP of $85 \mathrm{~mm} \mathrm{Hg}$ (the second data set). Finally, the NE dose was tapered down over $40 \mathrm{~min}$ to achieve again MAP of $65 \mathrm{~mm} \mathrm{Hg}$ (the third data set).
Fig. 1. Study flow chart. MAP mean arterial pressure norepinephrine

down-titration

\section{$40-60 \mathrm{~min}$}

\section{MAP}

$65 \mathrm{mmHg}$

During the study fluid infusion rates were kept constant, no red blood cells were given and no nursing procedures were performed.

\section{Statistics}

All values shown are medians; $25^{\text {th }}$ and $75^{\text {th }}$ percentiles if not otherwise stated. The differences between the periods were analyzed by the Friedman Repeated Measures Rank Sign Analysis of Variance and a subsequent Dunn's test for multiple comparisons when appropriate. The patients served as their own controls. Statistical significance was considered at $\mathrm{p}<0.05$.

\section{Results}

The study was performed on the Day 1-5 of the intensive care unit stay. All patients required norepinephrine before the study to achieve mean arterial pressure $\geq 65 \mathrm{mmHg}$. No clinical adverse effects were registered during NE titration. NE up-titration (to approximately double dose vs. baseline in the majority of patients, Table 2) increased MAP to the predefined values in all patients (Fig. 2). We observed a mild increase in cardiac filling pressures (Table 2, Fig. 3). Systemic and pulmonary vascular resistances increased at a higher MAP. This response with a subsequent return to baseline values at NE down titration was uniform in all patients. The observed changes in heart rate and stroke volume did not reach statistical significance. Cardiac output (CO) increased or remained unchanged in 10 patients during NE up-titration (Fig. 4). In 2 patients (No. 5 and No. 10), we observed $\mathrm{CO}$ decrease at a higher MAP due to the worsening of mitral regurgitation (W vawe increase). Neither arterial lactate levels, nor mixed venous oxygen saturation changed during the study.

\section{Discussion}

The main finding of our study was that norepinephrine (NE) induced rise of systemic vascular 
Table 2. Results.

\begin{tabular}{|c|c|c|c|c|}
\hline & MAP 65 (1) & MAP 85 & MAP 65 (2) & $\mathbf{P}$ \\
\hline$N E$ dose $(\mu \mathrm{g} / \mathrm{kg} / \mathrm{min})$ & $0.13(0.10 ; 0.20)$ & $0.25(0.21 ; 0.34)^{*}$ & $0.14(0.12 ; 0.21)^{* *}$ & $<0.001$ \\
\hline Heart rate (b.p.m.) & $83(80 ; 102)$ & $90(81 ; 101)$ & $85(80 ; 100)$ & 0.49 \\
\hline$M A P(m m H g)$ & $67(65 ; 69)$ & $84(83 ; 85)^{*}$ & $67(66 ; 69)^{* *}$ & $<0.001$ \\
\hline$C V P(m m H g)$ & $12(11 ; 14)$ & $14(12 ; 15)$ & $12(10 ; 14)^{* *}$ & $<0.001$ \\
\hline PAOP (mmHg) & $19(18 ; 20)$ & $23(21 ; 28)^{*}$ & $19(17 ; 19)^{* *}$ & $<0.001$ \\
\hline$M P A P(m m H g)$ & $28(25 ; 30)$ & $33(29 ; 40)^{*}$ & $28(25 ; 32)^{* *}$ & $<0.001$ \\
\hline$S V R\left(d y n * s / \mathrm{cm}^{5} * m^{-2}\right)$ & $2441(2039 ; 2764)$ & $2893(2332 ; 3869)^{*}$ & $2423(2008 ; 2725)^{* *}$ & $<0.001$ \\
\hline$P V R\left(d y n * s / \mathrm{cm}^{5} * m^{-2}\right)$ & $340(244 ; 463)$ & $554(307 ; 748)^{*}$ & $396(233 ; 513)^{* *}$ & $<0.001$ \\
\hline Stroke volume $\left(\mathrm{ml} / \mathrm{m}^{2}\right)$ & $22(18 ; 25)$ & $21(18 ; 24)$ & $22(19 ; 25)$ & 0.98 \\
\hline Cardiac index $\left(\mathrm{l} / \mathrm{min} / \mathrm{m}^{2}\right)$ & $1.9(1.6 ; 2.3)$ & $1.9(1.6 ; 2.4)$ & $1.9(1.6 ; 2.4)$ & 0.90 \\
\hline $\operatorname{LVSWI}\left(\mathrm{gm} / \mathrm{m}^{2} / H R\right)$ & $15(11 ; 17)$ & $17(14 ; 22)^{*}$ & $14(12 ; 18)$ & $<0.05$ \\
\hline Lactate $(\mathrm{mmol} / \mathrm{l})$ & $1.54(1.43 ; 1.92)$ & $1.50(1.46 ; 1.85)$ & $1.40(1.31 ; 1.85)$ & 0.33 \\
\hline $\mathrm{SvO}_{2}(\%)$ & $64(62 ; 69)$ & $64(62 ; 67)$ & $65(62 ; 69)$ & 0.79 \\
\hline
\end{tabular}

MAP - mean arterial pressure, NE - norepinephrine, CVP - central venous pressure, PAOP - pulmonary arterial occlusion pressure, MPAP - mean pulmonary arterial pressure, SVR - systemic vascular resistance, PVR - pulmonary vascular resistance, LVSWI - left ventricular stroke work index, $\mathrm{SvO}_{2}-$ mixed venous hemoglobin oxygen saturation. $*$ vs. MAP $65(1), * *$ vs. MAP 85 , NS $=$ nonsignificant

resistance and mean arterial pressure (MAP within a normal range of $65-85 \mathrm{~mm} \mathrm{Hg}$ ) did not compromise cardiac output in the majority of our patients.

In our study, all patients were euvolemic (individual preload optimization before the study). During norepinephrine up-titration, we observed a mild increase in both central venous and pulmonary arterial occlusion pressures (PAOP) which may be explained by the rise in systemic venous tone and pulmonary vascular tone and partly by a shorter diastole due to a mild heart rate increase. Cardiac output remained unchanged despite the rise of both systemic and pulmonary vascular resistances presumably due to the combined effect of norepinephrine both on alfa- and beta-adrenergic receptors. Nevertheless, the hemodynamic response to increased MAP in our study might have been influenced by the treatment with positive inotropic drugs (dobutamine in moderate stable doses and/or levosimendan in previous days) and thus we cannot exclude that cardiac output changes could be different in patients without previous or concurrent inotropic support.

During NE up-titration, clinician may expect hemodynamic deterioration in patients with severe mitral regurgitation (MR), where norepinephrine induced rise in systemic vascular resistance may lead to cardiac output decrease (afterload mismatch). We observed a mild cardiac output decrease in two patients during NE up-titration due to the worsening of mitral regurgitation (W wave increase/appearance). One patient had moderate to severe MR (3/4) already at baseline, whereas the second patient had only mild MR (1/4) at baseline. Therefore, before norepinephrine up-titration, a caution is needed when MR is detected on echocardiography and/or $\mathrm{W}$ wave is present during pulmonary arterial occlusion pressure measurement.

It is assumed that certain subgroups of critically ill patients might benefit from higher organ perfusion pressures, at least during limited time periods (Albanese et al. 2004, Marik 2004). The optimal MAP value may differ in 1) different patient populations (e.g. preexisting arterial hypertension, when autoregulatory renal perfusion pressure is shifted to higher values), 2) various stages of critical illness, e.g. the early phase in patients after cardiopulmonary resuscitation, when higher cerebral perfusion pressure is required or during sepsis when vasoplegia and altered vascular reactivity may result in a shift of the autoregulatory threshold to higher values), and 3) various organs also depending on different degree of stenotic atherosclerotic process (Marik 2004, Tamborini et al. 2001, Koužecký et al. 2006, Martin et al. 1999).

In a healthy heart, coronary blood flow remains stable within a wide range of coronary perfusion pressures (50-70 to $110-130 \mathrm{~mm} \mathrm{Hg}$ ) (Bourdarias 1995, 


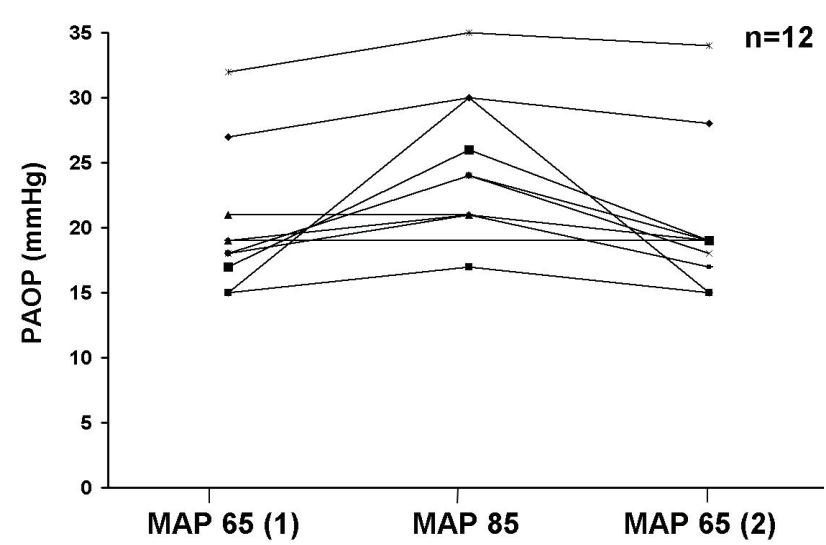

Fig. 3. Time course of pulmonary arterial occlusion pressure. PAOP - pulmonary arterial occlusion pressure, MAP - mean arterial pressure

Jones et al. 1995, Bellomo 2003). Norepinephrine leads to vasoconstriction of coronary arteries and coronary perfusion pressure (CPP) elevation (Heusch 1990). In experimental studies in sheep, short-term NE uptitration significantly increased coronary blood flow (Di Giantomasso et al. 2003, Bellomo 2003). In our study, we did not measure coronary blood flow directly. NE induced a significant MAP rise and a mild increase in PAOP, thus presumably also a mild increase of left ventricular end-diastolic pressure (LVEDP). Hence, we can assume that CPP (MAP-LVEDP) rose since the shortening of diastole (only mild heart rate increase) was not clinically significant (Vis et al. 1997). Importantly, the majority of our patients had successful percutanous coronary revascularisation with stent implantation before the study. We can only speculate that the effect of NE may be different in patients with persistent significant coronary artery stenosis, when the autoregulatory threshold of CPP may be shifted to higher values (Marik 2004).

\section{Study limitations}

Our study has certain limitations. First, the time periods for norepinephrine up- and down-titration were short and the increase of NE dose was relatively mild. Exposure to higher NE doses with more pronounced LV and RV afterload and/or heart rate increase might worsen cardiac performance due to the rise in myocardial oxygen demand or due to catecholamine-induced myocardial stunning (de Zeeuw et al. 2001, Bolli and Marban 1999). Moreover, using isolated rabbit hearts, Rump et al. (2002) demonstrated direct cardiotoxic effects of NE mediated by superoxide anion radicals depending on the dose of exogenous NE. In experimental studies it has also

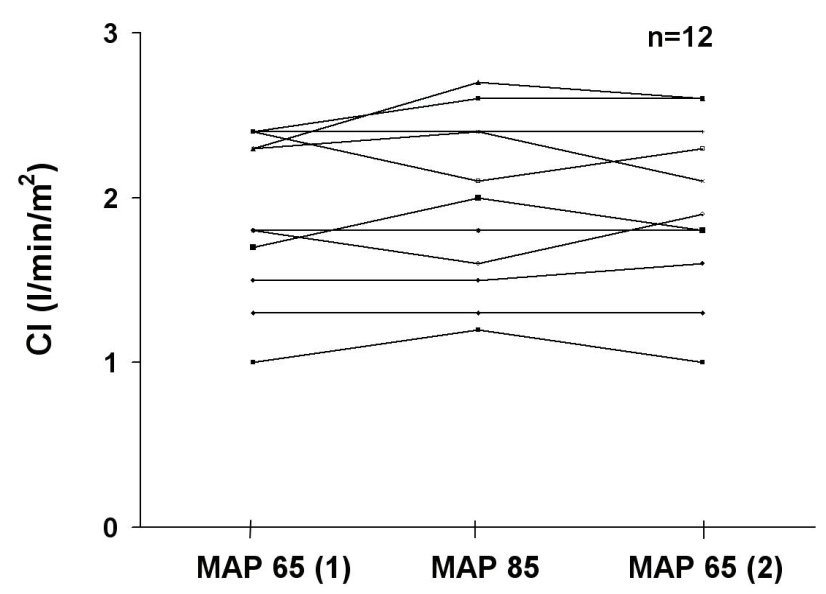

Fig. 4. Time course of cardiac index. CI - cardiac index, MAP mean arterial pressure

been shown, that long-term NE administration may induce cardiac fibrosis and/or left ventricular hypertrophy contributing to functional deterioration and heart failure exacerbation (Briest et al. 2001, Meier et al. 2007).

Second, our analysis was focused only on global hemodynamics and metabolism and not on regional hemodynamics. In previous studies, it has not been convincingly shown that higher MAP values improves regional blood flow (Zhang et al. 1997). In our recent experimental study in pigs with hyperdynamic septic shock, we have shown that neither intestinal macro- nor microcirculation was compromised by norepinephrine up-titration (Kroužecký et al. 2006). In the present human study, we found that the indices of global oxygen kinetics and metabolism were unaltered, but we cannot make any conclusion concerning regional blood flows and metabolism. Due to the short-term protocol of this study, we did not measure potential urine output changes.

\section{Conclusions}

We conclude that short-term norepinephrine dose up-titration leading to mild mean arterial pressure rise in euvolemic cardiogenic shock patients treated or pretreated by inotropes was tolerated well by the diseased heart. Our observation may be in favor of possible safe norepinephrine use in order to achieve a higher organ blood flow also in critically ill patients with compromised cardiac function under careful hemodynamic monitoring. Caution is needed in patients with mitral regurgitation. However, the impact of long-term norepinephrine infusion on cardiac function in this patient population requires further studies. 


\section{Conflict of Interest}

There is no conflict of interest.

\section{Acknowledgements}

Financial support by the cardiovascular research project of the Charles University Prague, No. MSM0021620817 „Invasive approach to myocardial salvage and regeneration".

\section{References}

ALBANESE J, LEONE M, GARNIER F, BOURGOIN A, ANTONINI F, MARTIN C: Renal effects of norepinephrine in septic and nonseptic patients. Chest 126: 534-539, 2004.

BELLOMO R: Noradrenaline: friend or foe? Heart Lung Circ 12: S42-48, 2003.

BOLLI R, MARBAN E: Molecular and cellular mechanisms of myocardial stunning. Physiol Rev 79: 609-634, 1999.

BOURDARIAS JP: Coronary reserve: concept and physiological variations. Eur Heart J 16: 2-6, 1996.

BOURGOIN A, LEONE M, DELMAS A, GARNIER F, ALBANESE J, MARTIN C: Increasing mean arterial pressure in patients with septic shock: Effects on oxygen variables and renal function. Crit Care Med 33: 780$786,2005$.

BRIEST W, HÖLZL A, RASSLER B, DETEN A, LEICHT M: Cardiac remodelling after long term norepinephrine treatment in rats. Cardiovasc Res 52: 265-273, 2001.

DE ZEEUW S, LAMERIS TW, DUNCKER DJ, HASAN D, BOOMSMA F, VAN DEN MEIRACKER AH, VERDOUW PD: Cardioprotection in pigs by exogenous norepinephrine but not by cerebral ischemia-induced release of endogenous norepinephrine. Stroke 32: 767-774, 2001.

Di GIANTOMASSO, MAY CN, BELLOMO R: Norepinephrine and vital organ blood flow during experimental hyperdynamic sepsis. Intensive Care Med 29: 1774-1781, 2003.

ĎURIŠOVÁ M, DEDÍK L, KRISTOVA V, VOJTKO R: Mathematical model indicates nonlinearity of noradrenaline effect on rat renal artery. Physiol Res 57: 785-788, 2008.

FRANCIS GS: Neurohumoral mechanisms involved in congestive heart failure. Am J Cardiol 55: 15A-21A, 1985.

HEUSCH G: Alpha-adrenergic mechanisms in myocardial ischemia. Circulation 81: 1-13, 1990.

JONES CJ, KUO L, DAVIS MJ, CHILIAN WM: Regulation of coronary blood flow: coordination of heterogeneous control mechanisms in vascular microdomains. Cardiovasc Res 29: 585-596, 1995.

KROUŽECKÝ A, MATĚJOVIČ M, RADĚJ J, ROKYTA R Jr, NOVÁK I: Perfusion pressure manipulation in porcine sepsis: effects on intestinal hemodynamics. Physiol Res 55: 527-533, 2006.

LEDOUX D, ASTIZ ME, CARPATI CM, RACKOW EC: Effects of perfusion pressure on tissue perfusion in septic shock. Crit Care Med 28: 2729-2732, 2000.

MARIK P: Renal dose norepinephrine. Chest 126: 335-337, 2004.

MARTIN C, VIVIAND X, AMAUD S, VIALET R, ROUGNON T: Effects of norepinephrine plus dobutamine or norepinephrine alone on left ventricular performance of septic shock patients. Crit Care Med 27: 1708-1713, 1999.

MATĚJOVIČ M: Noradrenaline and the kidney: foe, friend, or both? Intensive Care Med 31: 1476-1478, 2005.

MEIER H, BULLINGER J, DETEN A, MARX G, RABALD S, ZIMMER HG: Tissue inhibitor of matrix metalloproteinase-1 in norepinephrine- induced remodelling of the mouse heart. Cell Physiol Biochem 20: 825 836, 2007.

RUMP AFE, SCHIERHOLZ J, KLAUS W: Studies on the cardiotoxicity of noradrenaline in isolated rabbit hearts. Arzneim-Forsch/Drug Res 7: 543-551, 2002.

TAMBORINI G, MALTAGLIATI A, TRUPIANO L, BERNA G, SISILLO E, SALVI L, PEPI M: Lowering of blood pressure and coronary blood flow in isolated systolic hypertension. Coron Artery Dis 12: 259-265, 2001.

VIS MA, SIPKEMA P, WESTERHOF N: Modeling pressure-flow relations in cardiac muscle in diastole and systole. Am J Physiol 272: H1516-H1526, 1997.

ZHANG H, SMAIL N, CABRAL A, ROGIERS P, VINCENT JL: Effect of norepinephrine on regional blood flow and oxygen extraction capabilities during endotoxic shock. Am J Respir Crit Care Med 155: 1965-1971, 1997. 